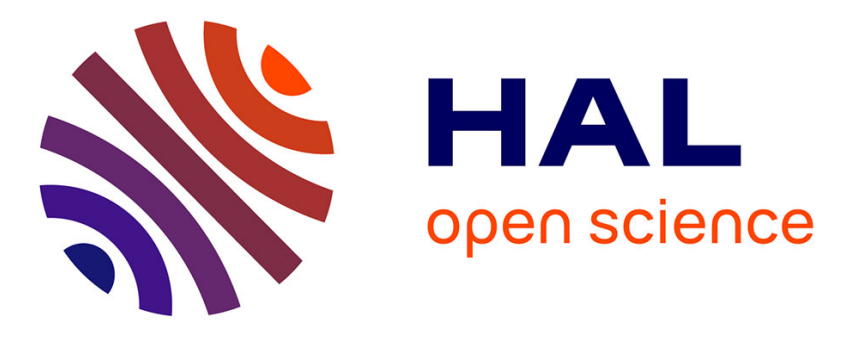

\title{
First detection and genome sequencing of SARS-CoV-2 in an infected cat in France
}

\author{
Corinne C. Sailleau, Marine Dumarest, Jessica Vanhomwegen, Manon \\ Delaplace, Valérie Caro, Aurélia Kwasiborski, Véronique Hourdel, Patrick \\ Chevaillier, Alix Barbarino, Loïc Comtet, et al.
}

\section{To cite this version:}

Corinne C. Sailleau, Marine Dumarest, Jessica Vanhomwegen, Manon Delaplace, Valérie Caro, et al.. First detection and genome sequencing of SARS-CoV-2 in an infected cat in France. Transboundary and emerging diseases, 2020, 67 (6), pp.2324-2328. 10.1111/tbed.13659 . hal-02874788

\section{HAL Id: hal-02874788 \\ https://hal.science/hal-02874788}

Submitted on 11 Sep 2020

HAL is a multi-disciplinary open access archive for the deposit and dissemination of scientific research documents, whether they are published or not. The documents may come from teaching and research institutions in France or abroad, or from public or private research centers.
L'archive ouverte pluridisciplinaire HAL, est destinée au dépôt et à la diffusion de documents scientifiques de niveau recherche, publiés ou non, émanant des établissements d'enseignement et de recherche français ou étrangers, des laboratoires publics ou privés.

$$
\text { Copyright }
$$


DR. CORINNE SAILLEAU (Orcid ID : 0000-0001-9077-6934)

Article type : Rapid Communication

Corresponding author mail id:- corinne.sailleau@anses.fr

First detection and genome sequencing of SARS-CoV-2 in an infected cat in France

C. Sailleau ${ }^{1}$, M. Dumarest ${ }^{1}$, J. Vanhomwegen ${ }^{2,3}$, M. Delaplace ${ }^{1}$, V. Caro ${ }^{2,3}$, A. Kwasiborski ${ }^{2}, 3$, V.Hourdel ${ }^{2,3}$ P.Chevaillier ${ }^{4}$, A. Barbarino ${ }^{5}$, L. Comtet ${ }^{6}$, P. Pourquier ${ }^{6}$, B. Klonjkowski ${ }^{1}$, J.C. Manuguerra ${ }^{2,3}$, S. Zientara ${ }^{1}$, S. Le Poder ${ }^{1}$.

1) UMR VIROLOGIE, INRAE, Ecole Nationale Vétérinaire d'Alfort, ANSES, Laboratoire de santé animale, Université Paris-Est, Maisons-Alfort, 94700, France.

2 Institut Pasteur, Laboratory for Urgent Response to Biological Threats, Environment and Infectious Risk Unit, Paris, France

${ }^{3}$ Institut Pasteur, The OIE Collaborating Centre for Detection and Identification in Humans of Emerging Animal Pathogens, Paris, France

${ }^{4}$ Clinique vétérinaire, Savigny sur Orge, France

${ }^{5} \mathrm{CHUVA}$ Ecole Nationale Vétérinaire d'Alfort, , Université Paris-Est, Maisons-Alfort, 94700, France

${ }^{6}$ IDVET, 310 rue Louis Pasteur - 34790 GRABELS

Corresponding author: Sophie Le Poder

This article has been accepted for publication and undergone full peer review but has not been through the copyediting, typesetting, pagination and proofreading process, which may lead to differences between this version and the Version of Record. Please cite this article as doi: 10.1111/TBED.13659

This article is protected by copyright. All rights reserved 
Short running title: Natural infection of a French cat by SARS-CoV-2

\section{Summary}

After its first description in Wuhan (China), SARS-CoV-2 the agent of coronavirus disease 2019 (COVID-19) rapidly spread worldwide. Previous studies suggested that pets could be susceptible to SARS-CoV-2. Here, we investigated the putative infection by SARS-CoV-2 in 22 cats and 11 dogs from owners previously infected or suspected of being infected by SARS-CoV-2. For each animal, rectal, nasopharyngeal swabs and serum were taken. Swabs were submitted to RT-qPCR assays targeting 2 genes of SARS-CoV-2.

All dogs were tested SARS-CoV-2 negative. One cat was tested positive by RT-qPCR on rectal swab. Nasopharyngeal swabs from this animal were tested negative. This cat showed mild respiratory and digestive signs. Serological analysis confirms the presence of antibodies against the SARS-CoV-2 in the both serum samples taken 10 days apart. Genome sequence analysis revealed that the cat SARS-CoV-2 belongs to the phylogenetic clade A2a like most of the French human SARS-CoV-2.

This study reports for the first time the natural infection of a cat in France (near Paris) probably through their owners. There is currently no evidence that cats can spread COVID-19 and owners should not abandon their pets or compromise their welfare.

Keywords : SARS-CoV-2; pets ; cats ; luminex ; ELISA ; next-generation sequencing

\section{Introduction}

Coronavirus disease 2019 (COVID-19) caused by the severe acute respiratory syndrome coronavirus 2 (SARS-CoV-2) was first reported in Wuhan (Zhou et al, 2020; Wu et al, 2020), China, and rapidly spread worldwide. Previous studies suggested cats could be a susceptible species to SARS-CoV-2 (Zhang, Q. et al 2020, Chi et al 2020). Two cases of natural infection in dogs, without symptoms, were also reported in Hong Kong (Sit el al, 2020). Only 4 naturally infected cats have been reported to date (promed posts or OIE notifications). Among them, two exhibited cough and another one mild respiratory and digestive 
signs. SARS-CoV-2 infection was also reported in lions and tigers in the zoo in New-York, suggesting susceptibility of wild Felidae to this virus.

We investigated the putative infection by SARS-CoV-2 in cats and dogs from owners previously confirmed or suspected of being infected with COVID-19. Among them, one cat was tested positive by RT-qPCR on rectal swabs and serological analysis. The SARS-CoV-2 genome has been almost completely sequenced, and clusters with the French human sequences circulating among infected humans.

\section{Cohort - Sample collection}

A total of 22 cats and 11 dogs were sampled from veterinary clinics (in the Paris or Grand Est area) or the pet hospital of the Alfort veterinary school (CHUVA) in April 2020. All animals selected in this study showed clinical signs and belonged to owners confirmed or suspected of having COVID-19. The clinical pictures reported were anorexia, hyperthermia, lethargy, diarrhoea, cough, bronchopneumonia, respiratory distress. Cats and dogs ranged in age from 6 months to 16 years and 2.5 months to 14 years respectively.

Serum, nasopharyngeal (NP) and rectal (R) swabs were collected and stored at $-80^{\circ} \mathrm{C}$ until analysis. The time between sampling and the appearance of the first symptoms ranged from 0 to 21 days depending on the animals.

\section{RNA Extraction and RT-PCR}

NP and R swabs were resuspended by vortexing in $300 \mu \mathrm{L}$ of PBS. Total RNA was extracted from $140 \mu \mathrm{L}$ of supernatant using either the robotic workstation 'QIAcube' and the QIAamp Viral RNA kit (Qiagen; reference: 52906) or robotic method based on magnetic beads (KingFisher with the MagVet Universal Isolation kit) according to the manufacturer's instructions and the protocols classically used in our laboratory for other viruses such as Foot-and-Mouth Disease, Bluetongue or West Nile viruses. Five $\mu \mathrm{L}$ of eluted RNA were used for the RT-qPCR assay using the commercial kit Bio-T kit ${ }^{\circledR}$ Covid-19 (BioSellal Dardilly -France))targeting the E gene.

At the same time, all the rectal swabs were tested by RT-qPCR targeting the feline coronavirus (FCoV) using the protocol previously described (D'Orengiani et al, 2015).

SARS-CoV-2 confirmatory tests were carried out in the OIE collaborating Centre in Institut Pasteur using the real-time duplex RT-PCR (targeting two regions of the RdRP gene) developed by the French National 
Reference Centre for Respiratory Viruses and the real-time RT-qPCR (targeting the E gene) from the Charité protocol (see WHO Coronavirus disease COVID-19 technical guidance: Laboratory testing for 2019nCoV in humans, available from https://www.who.int/docs/defaultsource/coronaviruse/whoinhouseassays.pdf).

\section{Next-generation sequencing}

Specimen RNA pre-treatment and Amplicon-based protocol. The ProtoScript II First Strand cDNA synthesis kit (New England Biolabs, USA) was used to obtain single strand cDNA from SARS-CoV-2-positive specimen RNA extract using random hexamers. For the multiplex PCR approach, the general method of multiplex PCR was applied using Version3 of amplicon sets, as described in (https://artic.network/ncov2019). After 40 rounds of amplification, the PCR products were cleaned-up with AMPure XP magnetic beads (Beckman Coulter, USA) and quantified with the Qubit 2.0 fluorometer (ThermoFisher Scientific, USA).

Illumina iSeq100 system sequencing. The sequencing-ready library was prepared using the Nextera DNA Flex Prep kit (Illumina, USA). The library was qualified on an Agilent Technologies 2100 Bioanalyser using a high-sensitivity DNA chip following the manufacturer's instructions. The resulting library was sequenced using the iSeq100 ${ }^{\text {TM }}$ System (Illumina, USA), generating $2 \times 150$ bp read length data.

Illumina bioinformatics workflow. To remove low quality reads, trim off low-quality and contaminant residues and filter out duplicated reads, fqCleaner v.0.5.0 was used, with Phred quality score of 28. Filtered reads were mapped against SARS-CoV-2 reference (NC_045512) using Burrows-Wheeler Aligner MEM algorithm (BWA-MEM) (v0.7.7). SAMtools were used to sort BAM files, to generate alignment statistics and to obtain consensus sequence.

\section{Serological analysis}

\section{- microsphere immunoassay- MIA-« luminex »}

Serum samples were tested using a microsphere immunoassay (MIA) for the presence of specific immunoglobulins against 5 coronavirus antigens: the SARS-CoV-2, SARS-CoV, NL63 and $229 \mathrm{E}$ nucleoproteins (NP) and the MERS-CoV spike (S) protein. Distinct MagPlex microsphere sets (Luminex Corp., USA) were bound to the recombinant viral proteins using the amine coupling kit (Bio-Rad Laboratories, USA) according to manufacturers' instructions. The MIA procedure was performed as described previously with minor modifications (Vanhomwegen et al., 2017). Briefly, microsphere mixtures

This article is protected by copyright. All rights reserved 
were sequentially incubated in the dark under constant shaking with a 1:400 dilution of serum samples, $4 \mu \mathrm{g} / \mathrm{mL}$ biotinylated protein A (Thermo Fisher Scientific, USA) and $2 \mu \mathrm{g} / \mathrm{mL}$ streptavidin-R-phycoerythrin (Life technologies, USA). After the final incubation, the median fluorescence intensity (MFI) of each antigen-coupled microsphere set was quantified for each sample using a BioPlex 200 instrument (Bio-Rad Laboratories). The seropositivity cut-off value was calculated for each antigen as three standard deviations above the mean MFI of ten negative control sera (sampled before 2019).

\section{- Serological analysis (ELISA)}

Cats sera were tested by an adapted version of the ID screen ${ }^{\circledR}$ SARS-CoV-2-N IgG indirect ELISA (IDvet, Grabels, France). The kit specifically detects anti-SARS-CoV-2 nucleocapsid antibodies. For this study, the conjugate supplied in the kit was changed to a multi-species conjugate to detect cats antibodies. Briefly, samples to be tested were diluted 1:20 in the sample dilution buffer and incubated for $45 \mathrm{~min}$ at room temperature. After washes, the multi-species conjugate, diluted in conjugate buffer, was added for 30 minutes at room temperature. The TMB substrate was added after the final washes, and the optical densities read at $450 \mathrm{~nm}$ after addition of stop solution. Calculation of ELISA cut-off value was established by the analysis of 16 negative cat control sera (sampled before 2019). The mean $(x)$ and standard deviation (SD) of these 16 negative. The cut-off value was taken as $x+3 S D$. OD values above this value were considered positive.

\section{Results and discussion}

All dogs were found SARS-CoV-2 PCR negative. Out of the 22 cats tested, one was SARS-CoV-2 Rt-qPCR positive with a Ct of 29 from the rectal swab whereas nasopharyngeal swabs were negative. Another cat was found positive for the feline coronavirus (Ct 25.6).

The RNA was sent to Institut Pasteur for confirmation of SARS-CoV-2 and was confirmed PCR positive (CT 43, 30 and 32 with RdRp IP2, RdRp IP4 and E genes).

The rectal swab extract found positive by RT-qPCR was subjected to targeted iSeq100 system sequencing, and the run produced $3,858,853$ raw reads with a Phred quality score of 28 . By using BWA-MEM, the mapping against SARS-CoV-2 reference (NC_045512) allowed the retrieval of high quality viral genomic sequences, with a read depth of 30x, covering around $94 \%$ of the full length genome (sequence data has been deposited in GISAID with accession number EPI_ISL_437349).

This article is protected by copyright. All rights reserved 
Sequence analysis of the cat SARS-CoV-2 genome showed that it belongs to the Nextstrain (https://nextstrain.org/) proposed phylogenetic clade A2a, which is consistent with the phylogenetic analysis of the recent human French SARS-CoV-2. Compared to the Wuhan reference sequence, and on the basis of the $94 \%$ covered genome, the cat SARS-CoV-2 sequence displays eight nucleotide mutations, with two not yet described in ORF1ab (T10800C and A13394G). Among these mutations, five have led to amino-acids mutations, of which four in ORF1ab (T265I, I3512T, K4377E and $\mathrm{P} 4715 \mathrm{~L})$. It should also be noted that the cat SARS-CoV-2 genome presents the amino-acid mutation D614G in the Spike glycoprotein, specific of the clade A2 and encompassing most of the French SARS-CoV2 sequences (figure 1 ).

The confirmed positive cat was a 9-year-old female of European breed. It has been hospitalised on 13 April due to anorexia, vomiting and cough. These clinical signs occurred 17 days after her owner has been sick from COVID-19. The first samples (NP and R swabs, serum) were taken on 17 of April, eight days after the beginning of symptoms. A second set of samples was taken on cat that was healed (on 27 April) and the PCR results were negative for both swabs. These results suggest a rapid clearance of the virus from the intestinal tract, which is consistent with experimental infection of cats suggesting presence of infectious virus only until day 6 post-infection (Shi et al, 2020). Serological analysis by MIA showed a strong positive signal against SARS-CoV-2 for the two sera taken at 10 days apart (figure 2). The value of fluorescence emitted was 36 times higher than the average of the values emitted by control cats (sera sampled before the appearance of COVID-19). A cross-reactivity was observed with the N SARS-CoV antigen but given the high sequence identity between the two antigens, this result was expected. Both sera were also confirmed positive by ELISA. The mean value of the sixteen cats sampled before 2019 and the apparition of the SARS-CoV-2 virus gave a mean OD value of 0,074 , with a standard deviation of 0,0116 . Therefore, the cut-off was OD450nm : 0,109 (mean of negative values $+3 x$ SD). The two sera taken at 10 days apart gave a mean value of 0.682 and 0.356 respectively. These OD values, clearly above the standard cut-off criteria (OD450 $\mathrm{nm} \mathrm{0,109),} \mathrm{reveal} \mathrm{the} \mathrm{presence} \mathrm{of} \mathrm{specific} \mathrm{antibodies} \mathrm{to} \mathrm{the} \mathrm{SARS-}$ CoV-2. The serological results (MIA + ELISA) confirm that the cat was able to replicate SARS-CoV-2.

Cats had already been tested positive for the SARS-CoV-2 in the United States, Belgium, Hong Kong and Wuhan, China, where outbreaks occurred. In the cases reported in Belgium and in the USA, clinical signs were reported (diarrhoea, vomiting and breathing difficulty for the first and respiratory illness for the second) whereas no clinical sign was detected in Hong Kong. Experimentally, pulmonary lesions have been described in young cats (Shi at al, 2020). Serological tests carried out on cats in Wuhan demonstrated that that SARS-CoV-2 has infected cat populations in Wuhan during the outbreak (15 of 102 tested were positive in ELISA and 11 had neutralizing antibodies with titers ranging from 1/20 to 1/1080). The highest

This article is protected by copyright. All rights reserved 
titers correspond to cats living with owners with confirmed COVID-19 infection. No information on clinical signs in any of the tested cats is available. Recently, Halfmann et al (2020) showed the possible transmission of SARS-CoV-2 between domestic cats in close contact. In this study, the virus could be detected only in feces. In other natural infections (the two cats in New York for instance), the virus was detected only in naso-pharyngal swabs whereas for the cat described in Belgium, the virus was found in the stool and vomits. The localisation of the virus may vary during the clinical phase of the disease or may depend on the clinical form. The cat described in this study and this from Belgium developed digestive symptoms which could explain the presence of the virus in feces whereas both cats in New York developed only respiratory symptoms. Further studies are needed to confirm the value of rectal versus nasopharyngeal sampling for better detection of the virus in infected animals and right now it would be better to investigate both samples.

As in previously identified cases worldwide, the French cat was living with a person who was confirmed positive for COVID-19. However, a previous study carried out on the animals of veterinary students of the Alfort veterinary school (Enva, Maisons Alfort), also infected by SARS-CoV-2 suggested that cats and dogs are not easily infected by the SARS-CoV-2, even when they are in close contact with infected owners (Temmam et al, 2020). Large serological analyses will be necessary to evaluate the real circulation of the SARS-CoV-2 among pets.

Concerning the infection of dogs with SARS-CoV-2, two cases have been reported in Honk- Kong in February and March 2020 (Sit, et al, 2020). In both cases, dogs were placed under quarantine after their owner was hospitalised due to COVID-19. Samples taken from the dogs were tested positive for SARSCoV-2 by RT-PCR and serological analysis. Both animals did not exhibit any specific clinical signs. Shi et al, 2020 have shown by experimental infection that dogs have low susceptibility to SARS-CoV-2. In our investigations, none of the 11 dogs tested were PCR positive despite having been in close contact with their infected owners and exhibiting clinical signs.

Even though the risk of transmission of the SARS-CoV-2 to the cat appears to be low, the authors therefore advise people with COVID-19 to limit close contact with their cats, without compromising their welfare.

\section{Acknowledgements}

The authors thank the veterinary practitioners for contributing to this study, the AFVAC (Association Française des Animaux de Compagnie) and the SNVEL of region lle de France for their help. We are also

This article is protected by copyright. All rights reserved 
grateful to Dr Meriadeg Le Gouil and Pr Astrid Vabret for helpful discussions. We thank Damien Hoinard for the technical support provided for the serological analysis.

\section{Ethical statement}

The authors confirm that the ethical policies of the journal, as noted on the journal's author guidelines page, have been adhered to and the appropriate ethical review committee approval has been received. The Directive 2010/63/EU was followed.

\section{Data availability Statement}

The data that support the findings of this study are available from the corresponding author upon reasonable request.

\section{Conflict of Interest Statement}

The authors declare that there they have no conflict of interests regarding the publication of this study.

\section{References}

Halfmann, P.J., M. Hatta, S. Chiba, T. Maemura, S. Fan, M. Takeda, N. Kinoshita, S.I. Hattori , Y. SakaiTagawa, K. Iwatsuki-Horimoto, M. Imai, Y. Kawaoka, 2020: Transmission of SARS-CoV2 in Domestic Cats. The New England journal of medicine, doi: 10.1056/NEJMc2013400.

d'Orengiani A.L., L. Duarte, N. Pavio, S. Le Poder, 2015 : Characterisation of different forms of the accessory gp3 canine coronavirus type I protein identified in cats. Virus Res. 202, 160-7

Shi, J., Wen Z, Zhong G and et. al., Susceptibility of ferrets, cats, dogs, and other domesticated animals to SARS-coronavirus 2 Science, [published online ahead of print, 2020 Apr 8] 2020;eabb7015. doi:10.1126/science.abb7015 
Sit, T.H.C., C.J. Brackman, Ip SM, K.W.S. Tam, P.Y.T. Law, To EMW, V.Y.T. Yu, L.D. Sims, D.N.C. Tsang, D.K.W. Chu, Perera RAPM, Poon LLM, M. Peiris. Infection of dogs with SARS-CoV2. Nature. 2020 May 14. doi: 10.1038/s41586-020-2334-5. [Epub ahead of print

Temmam, S., A. Barbarino, D. Maso, S. Behillil, V. Enouf, C. Huon, A. Jaraud, L. Chevallier, M. Backovic, P. Pérot, P. Verwaerde, L. Tiret, S. Van der Werf and M. Eloit, 2020: Absence of SARS-CoV-2 infection in cats and dogs in close contact with a cluster of COVID-19 patients in a veterinary campus. bioRxiv 2020.04.07.029090; doi: https://doi.org/10.1101/2020.04.07.029090

Vanhomwegen, J., C. Beck, P. Despres, A. Figuerola, R. Garcia, S. Lecollinet, M. Lopez-Roig, J. C. Manuguerra and J. Serra-Cobo, 2017: Circulation of Zoonotic Arboviruses in Equine Populations of Mallorca Island (Spain). Vector Borne Zoonotic Dis, 17, 340-346.

Wu, F., S. Zhao, B. Yu, Y. M. Chen, W. Wang, Z. G. Song, Y. Hu, Z. W. Tao, J. H. Tian, Y. Y. Pei, M. L. Yuan, Y. L. Zhang, F. H. Dai, Y. Liu, Q. M. Wang, J. J. Zheng, L. Xu, E. C. Holmes and Y. Z. Zhang, 2020: A new coronavirus associated with human respiratory disease in China. Nature, 579, 265-269.

Zhang, Q., H. Zhang, K. Huang, Y. Yang, X. Hui, J. Gao, X. He, C. Li, W. Gong, Y. Zhang, C. Peng, X. Gao, H. Chen, Z. Zou, Z. Shi and M. Jin, 2020: SARS-CoV-2 neutralizingserum antibodies in catsV: A serological investigation. BioRxiv, 2020.04.01.021196.

Zou, L., F. Ruan, M. Huang, L. Liang, H. Huang, Z. Hong, J. Yu, M. Kang, Y. Song, J. Xia, Q. Guo, T. Song, J. He, H. L. Yen, M. Peiris and J. Wu, 2020: SARS-CoV-2 Viral Load in Upper Respiratory Specimens of Infected Patients. The New England journal of medicine, 382, 1177-1179

Figure 1. Phylogenetic analysis of French cat SARS-CoV-2 genome sequence. Multiple sequence alignment of 43 SARS-CoV-2 genomes (available in GISAID database) was obtained using MAFFT version 7.023b (Katoh and Standley 2013). The unrooted phylogenetic tree was constructed using RAxML (Stamatakis et al 2008) with general time-reversible plus gamma distribution substitution model and a rapid bootstrap (i.e. model $G T R+I+G$, bootstrap $=1000$ ). Nextstrain clades are indicated next to the corresponding nodes and branches are colored distinctly.

Figure 2. Comparison of SARS-CoV-2 antibody levels detected in the acute and convalescent serum samples of the SARS-CoV-2 positive cat and a panel of 10 negative control sera using a pentaplex 
Multiplex Immunoassay (MIA). The pentaplex MIA comprised a mixture of microspheres coupled to the nucleoproteins (NP) of SARS-CoV-2, SARS-CoV, 229E and NL63 and the spike protein (S) of MERS-CoV. The relative antibody levels in the serum samples are expressed as median fluorescence intensities (MFI).

This article is protected by copyright. All rights reserved 

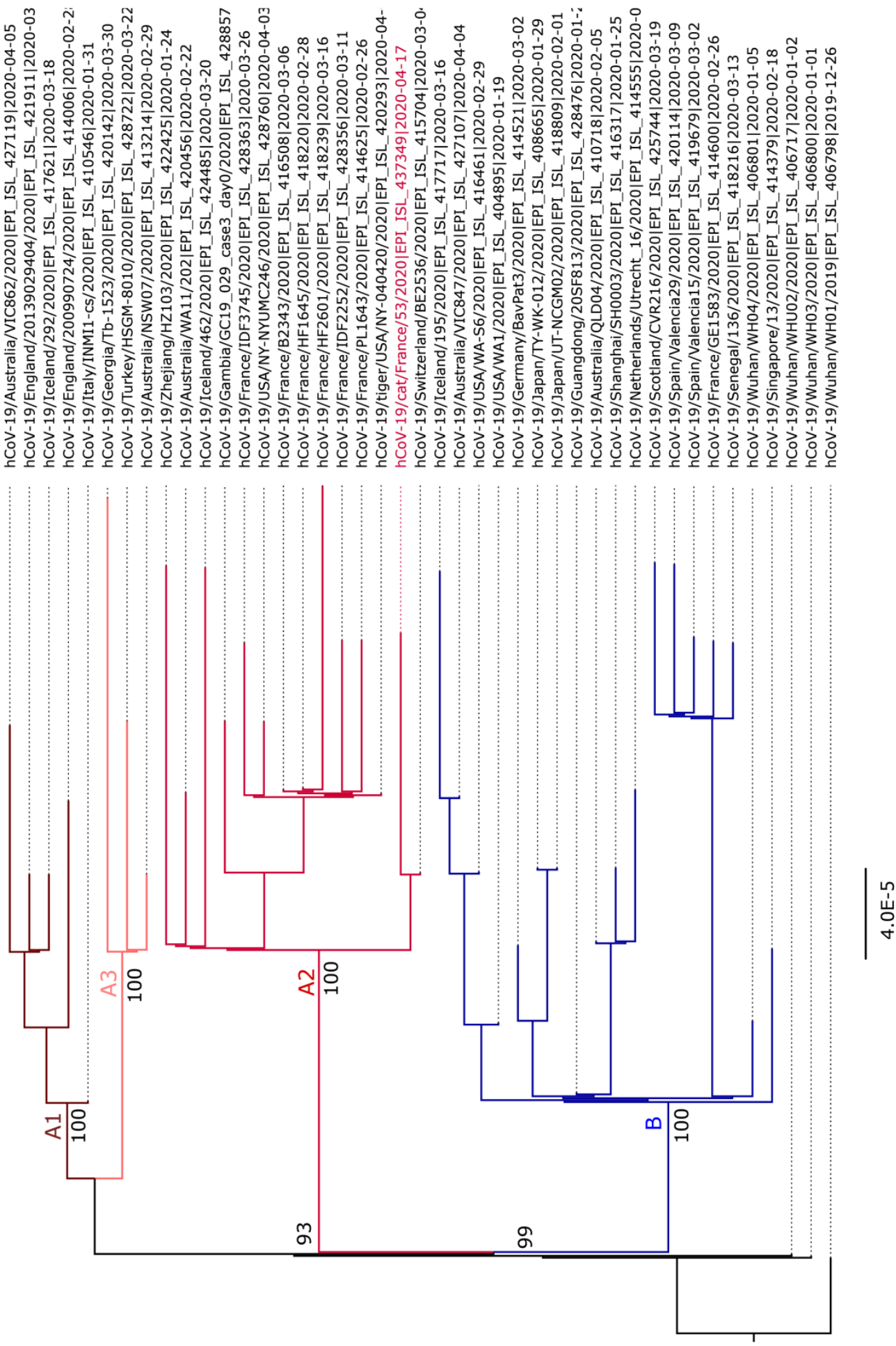

tbed_13659_f1.tif 

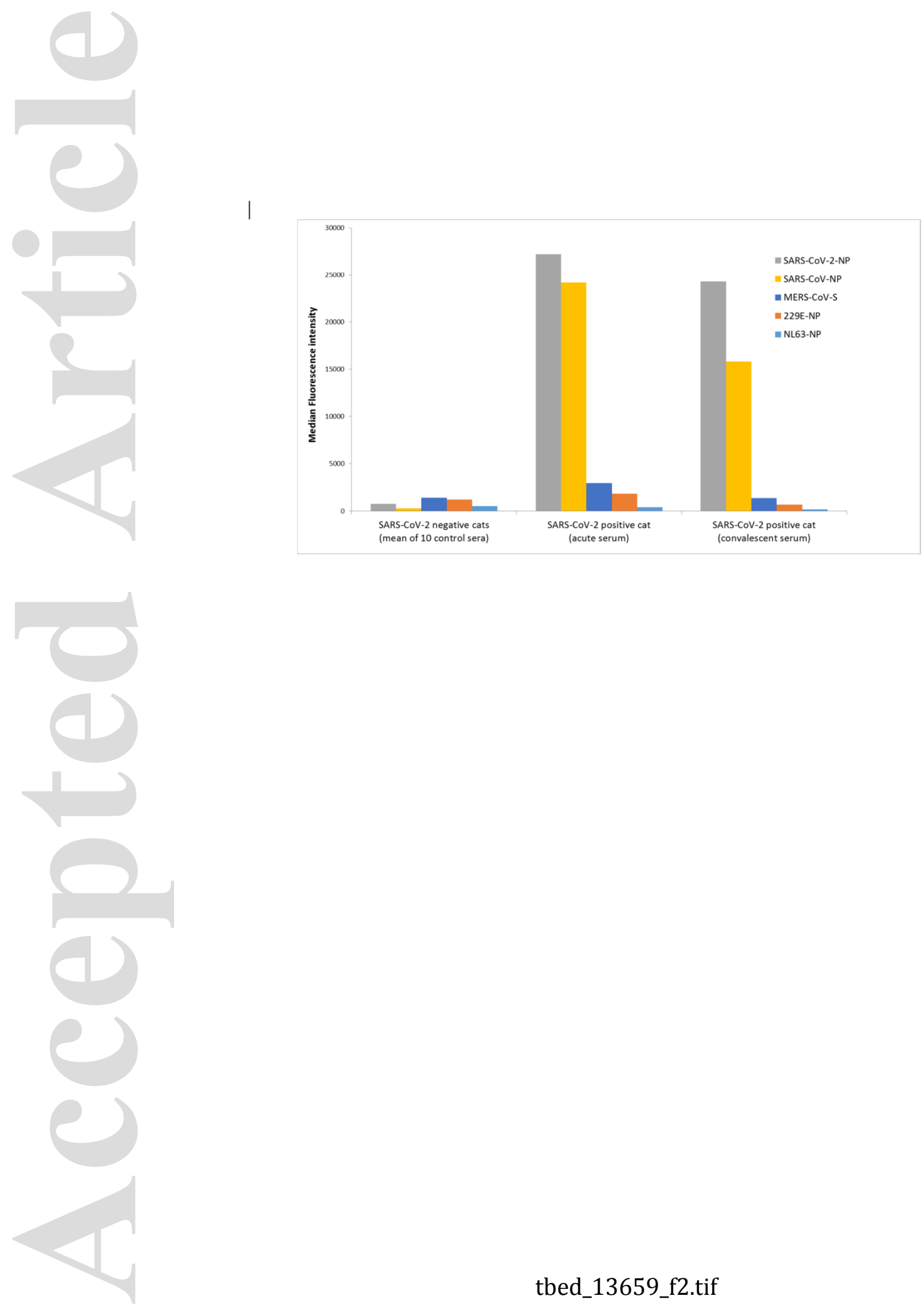

tbed_13659_f2.tif

This article is protected by copyright. All rights reserved 